\title{
Effectiveness of home respiratory polygraphy for the diagnosis of sleep apnoea and hypopnoea syndrome
}

\author{
Juan F Masa, ${ }^{1,2}$ Jaime Corral, ${ }^{1,2}$ Ricardo Pereira, ${ }^{1,2}$ Joaquin Duran-Cantolla, ${ }^{2,3}$ \\ Marta Cabello, ${ }^{4}$ Luis Hernández-Blasco, ${ }^{5}$ Carmen Monasterio, ${ }^{6}$ Alberto Alonso, ${ }^{2,7}$ \\ Eusebi Chiner, ${ }^{8}$ Manuela Rubio, ${ }^{1}$ Estefania Garcia-Ledesma, ${ }^{1}$ Laura Cacelo, ${ }^{3}$ \\ Rosario Carpizo, ${ }^{4}$ Lirios Sacristan, ${ }^{5}$ Neus Salord, ${ }^{6}$ Miguel Carrera, ${ }^{7}$ \\ José N Sancho-Chust, ${ }^{8}$ Cristina Embid, ${ }^{9}$ Francisco-José Vázquez-Polo, ${ }^{10}$ \\ Miguel A Negrín, ${ }^{10}$ Jose M Montserrat $^{2,9}$
}

${ }^{1}$ Pulmonary Service, San Pedro de Alcantara Hospital, Caceres, Spain

${ }^{2}$ CIBER de enfermedades respiratorias (CIBERES), Madrid, Spain

${ }^{3}$ Pulmonary Service, Txagoritxu Hospital, Vitoria, Spain

${ }^{4}$ Pulmonary Service, Valdecilla Hospital, Santander, Spain

${ }^{5}$ Pulmonary Service, General Universitario Hospital, Alicante, Spain

${ }^{6}$ Pulmonary Service, Belvitge Hospital, Barcelona, Spain

${ }^{7}$ Pulmonary Service, Son Dureta Hospital, Palma de Mallorca, Spain

${ }^{8}$ Pulmonary Service, San Juan Hospital, Alicante, Spain

${ }^{9}$ Pulmonary Service, Clinic Hospital, Barcelona, Spain ${ }^{10}$ Department of Quantitative Methods, Las Palmas de Gran Canaria University, Spain

\section{Correspondence to}

Dr Juan F Masa, San Pedro de Alcántara Hospital, Pablo Naranjo s/n, Cáceres 10003 Spain; fmasa@separ.es

Received 8 November 2010 Accepted 8 April 2011 Published Online First 20 May 2011

\section{ABSTRACT}

Introduction Home respiratory polygraphy (HRP) may be a cost-effective alternative to polysomnography for the diagnosis of sleep apnoea-hypopnoea syndrome (SAHS), but stronger evidence is needed. Normally, patients transport HRP equipment from the hospital to home and back, which may create difficulties for some patients.

Objectives To determine both the diagnostic efficacy and cost of HRP (with and without a transportation service moving the device and telematic transmission of data) in a large sample compared with in-hospital polysomnography.

Methods Patients suspected of having SAHS were included in a multicentre study (eight hospitals). They were assigned to home and hospital protocols in random order. Receiver operating characteristic curves were constructed for manual respiratory polygraphy scoring protocol and different polysomnographic cut-off points. Diagnostic efficacies for several polysomnographic cutoff points were explored and costs for two equally effective alternatives were calculated.

Results Of 366 randomised patients, 348 completed the protocol. The best receiver operating characteristic curve was obtained with a polysomnographic cut-off of the apnoea-hypopnoea index $(\mathrm{AHI}) \geq 5$. The sensitive HRP AHI cut-off point $(<5)$ had a sensitivity of $96 \%$, a specificity of $57 \%$ and a negative likelihood ratio (LR) of 0.07 ; the specific cut-off ( $>10$ ) had a sensitivity of $87 \%$, a specificity of $86 \%$ and a positive LR of 6.25 . The cost of HRP was half that of polysomnography. Telematic transmission costs were similar if the patients' costs were taken in to account.

Conclusion HRP is an alternative to polysomnography in patients with suspected SAHS. Telematic procedures may help patients with limited mobility and those who live a long way from the sleep centre.

\section{INTRODUCTION}

The prevalence of sleep apnoea-hypopnoea syndrome (SAHS) is about $2-5 \%$ in the adult population. ${ }^{1}$ A number of studies have shown associations with arterial hypertension, ${ }^{2}$ cardiovascular mortality ${ }^{3}$ and traffic accidents. ${ }^{4} 5$

Given this prevalence and the increasing awareness of SAHS in the medical community as well as in the general population, the demand for consultations and diagnostic studies has increased in
Key messages

What is the key question?

- Is home respiratory polygraphy a diagnostic alternative for sleep apnoea-hypopnoea syndrome?

What is the bottom line?

- Home respiratory polygraphy is a cost-effective alternative to polysomnogaphy for sleep apnoeahypopnoea syndrome diagnosis in a large multicentre study.

Why read on?

- Is a telematic approach with home respiratory polygraphy (from home to hospital) a costeffective alternative for diagnosis of sleep apnoea-hypopnoea syndrome?

recent years. Access to diagnostic testing is difficult, however, with long waiting lists. ${ }^{6} 7$ Therefore, there is growing interest in alternative diagnostic methods and approaches.

The classical method for diagnosis is the attended polysomnography (PSG), but it is time consuming and expensive. Home sleep studies with portable monitoring devices have been proposed to decrease costs and facilitate the diagnostic process. $^{89}$ The American Academy of Sleep Medicine $(\mathrm{AASM})^{9}$ recommended home diagnosis with portable monitoring devices in patients with a high pretest probability of moderate to severe SAHS, without significant comorbidities. A negative result should lead to a PSG. For this recommendation, the AASM proposed the use of a type 3 portable monitoring device ${ }^{10}$ which includes airflow, respiratory effort measured with bands, and pulse oxymetry recordings, which is a well known type of respiratory polygraphy. Studies performed with home respiratory polygraphy $(\mathrm{HRP})^{11-22}$ show methodological differences in design, protocol, HRP devices used, definitions of respiratory events, and cut-off points for SAHS diagnosis. In cost terms, only three of the studies mentioned included cost assessments $^{17-19}$ favourable to HRP, although other cost studies based on simulated models of hypothetical patient cohorts showed unfavourable cost effectiveness. ${ }^{23} 24$ In addition, the largest 
sample size was 89 patients $^{17}$ and there were no multicentre studies to minimise inter-centre variability.

To generate better evidence, a large multicentre, randomised controlled study with cost analysis is needed to compare the cost effectiveness of HRP and PSG. ${ }^{9}$

In clinical practice, patients transport HRP equipment from the hospital to their homes and return it to the hospital the following day. Some patients with SAHS have limited mobility because of comorbidities or extreme obesity. Consequently, establishing the effectiveness of a protocol that allows patients to remain at home using new communication technology would be beneficial for them, as well as for patients who live far from the sleep centre.

The authors carried out a multicentre, randomised, blinded crossover study of a large sample of patients with the objective of determining the diagnostic efficacy of HRP compared with PSG in the hospital setting, estimating the costs of HRP at the same level of diagnostic efficacy as PSG, with two approaches: patients transporting the device themselves; and the device being transported by trained personnel with a background in the telematic transmission of raw data.

\section{METHODS \\ Subjects}

We included patients between 18 and 70 years old, referred for pulmonary consultations to eight centres in Spain for suspected SAHS caused by snoring, observed apnoeas, sleepiness (Epworth sleepiness scale $>10$ ) or non-refreshing sleep. We excluded patients with other suspected sleep disorders, severe and unstable heart disease, who were unable to set up the HRP instrument in a trial or who refused to participate in the study. The study was approved by the ethics committees of the eight centres. Informed consent was obtained in writing from all patients.

\section{Protocol}

All patients underwent PSG (plus simultaneous respiratory polygraphy-SRP) and HRP with telematic transmission of the raw data file, in a random order (figure 1A). PSG, SRP and HRP scorings were done separately and the technicians and physicians were blinded to any identifying information about patients as well as any previous results. Once the first test had started, the second test was scheduled for within the next 3 days. Two HRP devices of the same model (BreastSC20; Breast Medical AB, Mölnlycke, Sweden) were used at each centre, one in the hospital and the other at the patient's home.

\section{Home respiratory polygraphy}

HRP measurements included oxygen saturation (model $8000 \mathrm{~J}$; Nonin Medical, Plymouth, Minnesota, USA), airflow through a nasal cannula, and thoracic and abdominal movements measured by piezoelectric bands (Pro-Tech reference 1295; Respironics, Pittsburgh, Pennsylvania, USA), which also measured body position. The HRP device collected this information in a data card. The authors developed software that was installed into a personal digital assistant (PDA) phone that automatically read the data file from the card, sent it by general packet radio service (GPRS) or universal mobile telecommunications system (UMTS) (depending on signal strength) and then deleted the file. The transmitted file was saved in a previously developed website.

The HRP device was moved by trained personnel from continuous positive airway pressure (CPAP) service companies in each hospital area, acting as specialised transport companies. Their job was to transport the device from one residence to another and, after 10 uses, return it to the hospital for servicing; and to carry out simple procedures to initialise the data card in the HRP and transfer the data card from the HRP to the PDA the following day.

All patients were instructed on home use of the HRP device by a technician in the hospital setting before randomisation. The patients' responsibilities were to set up and remove the HRP device, and to toggle the HRP device on and off to start and stop recording.

The hospital technician downloaded the data file from the website and scored the raw data using a manual scoring protocol.

\section{PSG in the hospital}

The polysomnographic studies were analysed manually at each participating centre, according to standard criteria. ${ }^{25} 26$ The neurological variables were EEG, electrooculogram and electromyogram. Flow tracing was provided by a nasal cannula and thoracoabdominal motion by piezoelectric bands. Oxygen saturation was measured with a finger-pulse oxymeter. The nasal cannula was split by a Y connection to PSG and SRP. Other SRP parameters were measured by independent signals. The scoring of respiratory events in SRP was done with the same criteria as for HRP.

\section{Definitions}

A valid PSG or HRP had at least 3 recorded hours. In addition, a valid HRP had at least $3 \mathrm{~h}$ of flow or band and oxymetry measurements for scoring. An invalid recording could be repeated up to two times.

For PSG, an apnoea was defined as the absence of airflow ( $\geq 90 \%$ reduction) for $\geq 10 \mathrm{~s}$ and a hypopnoea as a discernible airflow or band reduction ( $\geq 30 \%$ and $<90 \%$ ) of at least $10 \mathrm{~s}$ duration with a $\geq 3 \%$ drop in oxygen saturation or final arousal. $^{27} 28$ In the respiratory polygraphy, apnoea and hypopnoea were defined in the same way, but without the final arousal criteria for hypopnoea. The number of apnoeas and hypopneas was divided by the recording time for HRP and the sleep time for PSG

\section{Cost analysis}

A cost analysis was used for two equally effective alternatives. ${ }^{29}$ Figure $1 \mathrm{~B}$ presents the procedure for calculating the costs of both alternatives. Although the HRP device was moved by the transport company with telematic transmission of raw data in all patients, we also estimated the cost if the device had been transported by patients in the following cost groups:

\section{Test costs (PSG and HRP)}

Mean hospital costs included the following expenditures: personnel (technicians, physicians and secretaries), linear 5-year depreciation of equipment (taking into account the number of recordings done in this period in each hospital as output), fungible material, and the proportional burden of the sleep laboratory in the general budget of the hospital. For the telematic approach, the following items were added to the cost of HRP: the transportation company, hardware (PDA and data card), the proportional burden per hospital for software development, hosting and maintenance of websites, and phone data transmission. The useful lifetime of software and hardware was considered to be 5 years; consequently the cost for this period was evaluated, considering the number of recordings done in this period in each hospital as output. The cost of PSG and HRP was 
Figure 1 (A). General flow chart of the patients during the study; 359 patients completed both branches of the study. (B). More detailed study flow chart to calculate the costs for two alternatives. A decision node refers to alternatives by protocol, random nodes include alternatives determined at random and terminal nodes mark the end of the branch and additional costs. In the HRP branch five patients were lost without adding costs. Patients with invalid recordings underwent one or two new HRPs with two options: (a) patients with a new invalid recording or without a diagnosis (grey zone and false negative diagnosis) reached the terminal node of the HRP arm; the costs of the consequent full PSG and that of HRP repetitions were added to the final cost; (b) patients with a diagnosis (true positive or negative) reached the terminal node of the HRP branch and the costs of HRP and repetitions were included. Patients with a valid diagnosis by HRP finished the HRP arm and the HRP expenditure was added; both the HRP and PSG costs were included for patients without a diagnosis. In the PSG branch, two patients were lost to follow-up without additional costs. In patients with an invalid PSG, it was repeated and they reached the terminal node of this arm, with the cost of two PSGs added to total PSG costs. Patients with a valid PSG contributed the cost of one PSG. HRP, home respiratory polygraphy; PSG, polysomnography; SAHS, sleep apnoea and hypopnoea syndrome.

divided by the number of patients with a valid recording to obtain the cost per patient.

\section{Patients' costs for PSG and HRP}

The cost was calculated of moving from home to hospital and back, per kilometre. The cost per kilometre was calculated for each hospital. The totals of the patients' costs were divided by the number of patients with a valid recording to derive cost per patient.

Total cost

The total cost was the sum of test (PSG and HRP) and patient costs.

Costs of HRP and PSG for equal diagnostic efficacy

For HRP with the device moved by patients, the cost was the sum of the HRP cost and the following: the cost of PSG in patients with invalid recordings, after repetitions; the cost of repeated HRPs because of invalid recordings in patients with a valid final recording; the cost of PSG in patients with indeterminate results ('grey zone'); and the cost of PSG in patients with false negative and positive results. For telematic HRP, the cost was the sum of the HRP cost with the device being moved by patients and the following: the cost of PSG in patients with invalid telematic transmission after repetitions; and the cost of repeated HRPs caused by invalid telematic transmissions in patients with a valid final recording.

For PSG, we added the initial test cost to the cost of repeated PSGs due to invalid recordings.

The cost for equal diagnostic efficacy (HRP and PSG) was divided by the number of randomised patients who completed the protocol to calculate the cost per patient.
Patients' costs for equal diagnostic efficacy

This was the patient cost plus the burden of transportation caused by repetitions.

Total cost for equal diagnostic efficacy

This cost was the sum of test costs (PSG and HRP) and patient costs for equal diagnostic efficacy.

\section{Statistical analysis}

We performed a 'per protocol' analysis for the main variables: diagnostic efficacy and cost. To determine the agreement of apnoea-hypopnoea index (AHI) measurements between PSG and SRP and between PSG and HRP, Bland-Altman plots were used

Separate receiver operating characteristic (ROC) curves were constructed for HRP with the different polysomnographic AHI cut-off points $(\geq 5, \geq 10, \geq 15)$ for SAHS diagnosis to determine the best ROC curve based on area under the curve (AUC) measurements. To determine the exclusive (sensitive) and the confirming (specific) HRP cut-off points for SAHS diagnosis, we calculated sensitivity and specificity, negative (1-sensitivity/ specificity) and positive (sensitivity/1-specificity) likelihood ratios (LRs). In addition, the post-test probability of obtaining a true positive diagnosis when the test was positive or negative was calculated, based on the pretest probability (prevalence) and positive and negative LRs. ${ }^{8}$

\section{RESULTS}

\section{Diagnostic efficacy of HRP}

Initially, 377 patients were selected. Eleven were excluded (figure 1A). Of the 366 randomised patients, 18 could not produce a valid HRP and PSG (4.9\%). Four of these patients 
(three starting with HRP and one with PSG) did not come to their scheduled appointments. Another rejected the PSG test after a valid HRP and two rejected HRP after a valid PSG.

Of the 359 patients who completed the protocol (both branches of the study), PSGs were repeated once in nine patients $(2.5 \%)$, HRPs once in 51 patients (14\%), and an additional time in 34 patients. From a total of 85 HRP repetitions, 15 were due to the patients' failure to toggle the device on or off, 37 to invalid or poor quality time registers and 33 to deficient telematic transmission or transportation procedures. Finally, 11 patients could not produce a valid HRP after repetitions (three because of deficient telematic procedures).

The clinical and anthropometric characteristics of the 348 patients with valid PSG and HRP results are shown in table 1. The PSG, SRP and HRP recording times were 442.5 \pm 42.8 , $447.2 \pm 48$ and $428.3 \pm 81.9$ respectively. The PSG sleep time was $371.5 \pm 64.3$ and the valid time for SRP and HRP was $432.7 \pm 48.9$ and 404.1 1.1 respectively. Consequently, at least in part, AHI scores were lower for HRP and SRP than for PSG. The mean AHI was 38.3 \pm 28.5 for PSG, $34 \pm 27.2$ for SRP and 31.3 \pm 24.1 for HRP.

Figure 2 shows Bland-Altman plots for PSG and SRP, and for PSG and HRP. Better agreement with PSG was observed for SRP than for HRP.

Figure 3 shows the ROC curves for HRP with the different polysomnographic cut-off points $(\geq 5, \geq 10, \geq 15)$ for SAHS diagnosis. All AUCs were statistically significant $(p<0.001)$, expressing a high level of diagnostic accuracy. The best and worse AUCs were obtained using PSG cut-off points of $\mathrm{AHI} \geq 5$ and $\mathrm{AHI} \geq 15$ respectively.

Table 2 shows the diagnostic efficacy of the HRP. For a polysomnographic AHI cut-off point of $\geq 5$, the pretest probability (prevalence) was $90 \%$. For the same polysomnographic cut-off point, an $\mathrm{AHI}<5$ from HRP effectively excluded SAHS because the negative LR was 0.07 and the pretest probability would decrease from $90 \%$ to a post-test probability of 39\%. An acceptable HRP AHI cut-off point for confirming SAHS would be 10 , with a positive LR of 6.25 , and the pretest probability would increase from $90 \%$ to a post-test probability of $98 \%$. The percentage of patients with a positive result (true or false positives) for a HRP AHI $\geq 5$ would be $90 \%$ and $80 \%$ for a HRP $\mathrm{AHI} \geq 10$ (table 2). Therefore, the indeterminate results (grey zone) should represent $10 \%$ of patients.

For a polysomnographic AHI cut-off point $\geq 10$, the pretest probability was $82 \%$ (table 2 ). For the same polysomnographic cut-off point, an AHI $<5$ from HRP effectively excluded SAHS

Table 1 Characteristics of the studied population $(\mathrm{N}=348)$

\begin{tabular}{ll}
\hline & Mean (SD) \\
\hline Men, \% & 75.6 \\
Age, years & $48.7(11.8)$ \\
Body mass index & $31.0(6.6)$ \\
Subjective sleep time/day, h & $6.9(1.4)$ \\
Subjective nap time, h & $0.5(0.6)$ \\
Epworth sleepiness scale & $11.6(5.0)$ \\
Alcohol, g & $8.9(22.3)$ \\
Smokers, \% & 23.9 \\
Hypertension, \% & 30.7 \\
Cardiovascular events, \% & 5.6 \\
Systolic pressure, mm Hg & $130.5(17.2)$ \\
Diastolic pressure, mm Hg & $76.0(12.1)$ \\
Home to hospital distance, km & $20.6(33.0)$ \\
Starting home, \% & $47.7 \%$ \\
\hline
\end{tabular}

because the negative LR was 0.08 and the pretest probability would decrease from $82 \%$ to a post-test probability of $27 \%$. In practice, the HRP confirmation cut-off point should be $\geq 20$, resulting in a positive LR of 7.10 and a post-test probability of $97 \%$. The grey zone would be $20 \%$.

For a polysomnographic AHI cut-off point $\geq 15$, the pretest probability was $75 \%$ (table 2 ). For the same polysomnographic cut-off point, an AHI $<10$ from HRP would effectively exclude SAHS because the negative LR was 0.10 and the pretest probability would decrease from $75 \%$ to a post-test probability of $23 \%$. Effective HRP confirmation cut-off points should be $\geq 25$, resulting in a positive LR of 8.36 and post-test probability of $96 \%$. The grey zone would be $28 \%$.

\section{Cost analysis}

Table 3 shows the cost per patient, from the eight hospitals, of PSG and HRP with and without telematic transfer for the calculated cost groups. Figure 4 shows the percentages of total HRP costs and their distribution into cost groups related to
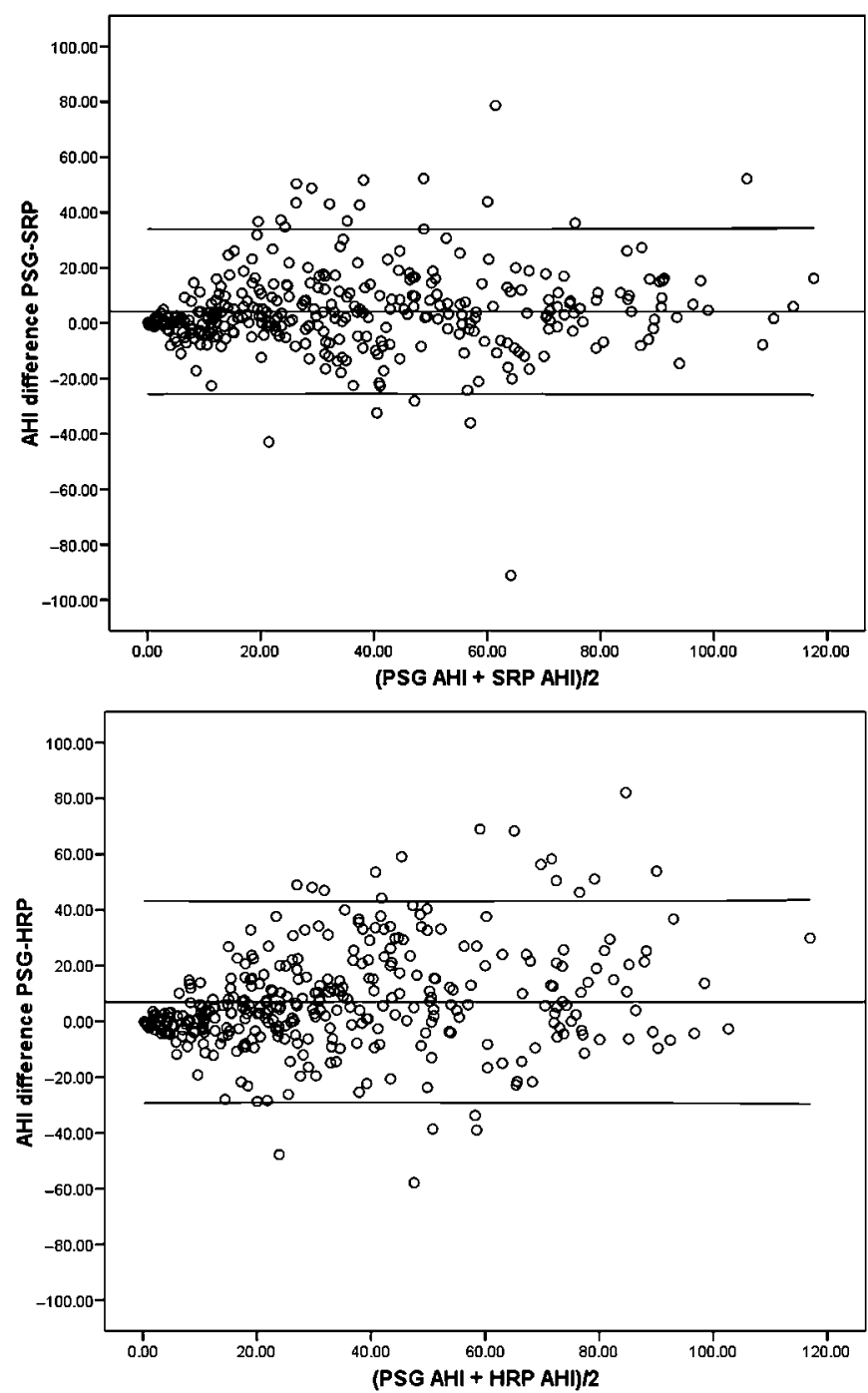

Figure 2 Mean AHI versus difference in AHI between PSG and SRP and between PSG and HRP (Bland-Altman plots). Central lines represent mean values and upper and lower lines represent agreement limits (two SDs). AHI, apnoea-hypopnoea index; HRP, home respiratory polygraphy; PSG, polysomnography; SRP, simultaneous respiratory polygraphy. 

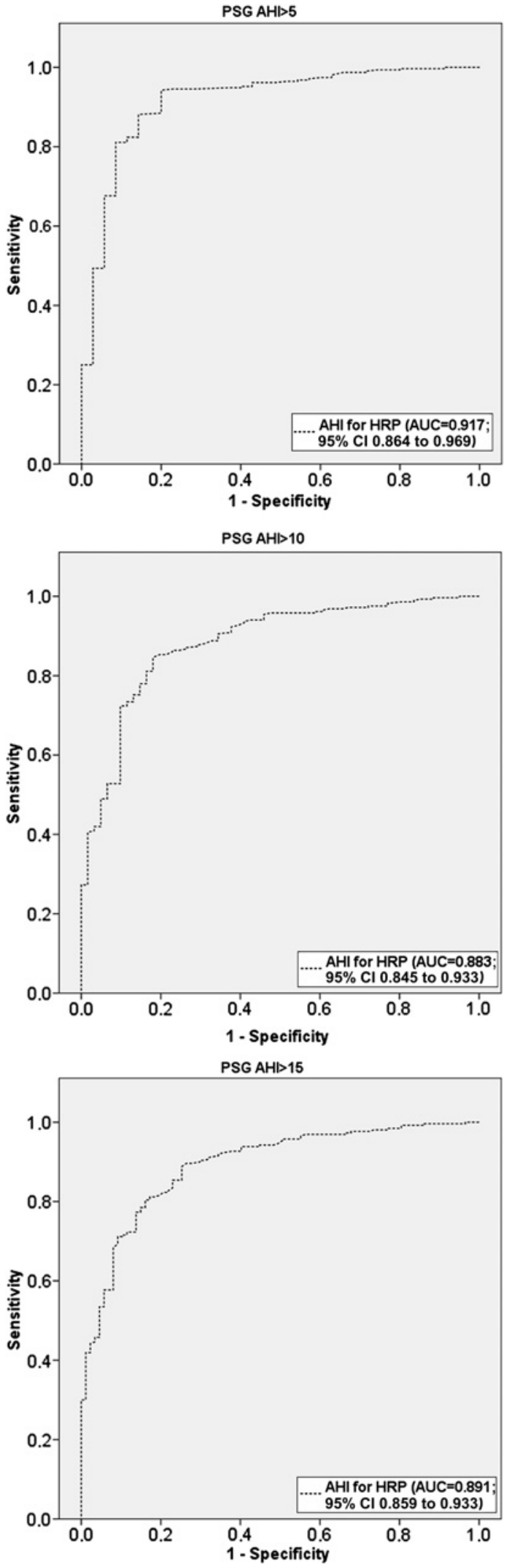

Figure 3 ROC curves for the home respiratory polygraphy scoring based on the three polysomnographic cut-off points of sleep apnoea and hypopnoea syndrome diagnosis $(\geq 5, \geq 10, \geq 15)$. AHI, apnoeahypopnoea index; AUC, area under the curve; HRP, home respiratory polygraphy; PSG, polysomnography; ROC, receiver operating characteristics. polysomnographic cost. The cost of HRP was six times lower than PSG when patients transported the device and four times lower using the telematic approach. The latter was similar to HRP transported by patients, if patient costs were taken into account.

To estimate the cost of HRP for diagnostic efficacy equal to PSG the most unfavourable polysomnographic cut-off point $(\mathrm{AHI} \geq 15)$ was chosen and an HRP AHI cut-off point to effectively exclude $(<10)$ and to effectively confirm $(\geq 25)$ SAHS, with indeterminate scores (cases needing further PSG assessment) for 28\% (table 2). The cost of achieving an HRP efficacy equal to that of PSG (without the telematic approach) was three times higher than the test cost without equal efficacy, but half the cost of PSG. The cost of HRP (with the telematic approach), at this efficacy level, was $€ 42(15 \%)$ more expensive than HRP transported by patients. If we added the patient costs, however, the total cost for equal diagnostic efficacy was similar.

\section{DISCUSSION}

To our knowledge, this study has the largest sample of all available studies of the diagnostic efficacy of portable monitoring. The study is four times larger than any previously published HRP study ${ }_{1}^{17}$ as well as being the only multicentre study. The principal results are that HRP can exclude and confirm the diagnosis of SAHS; and for equal diagnostic efficacy, the cost of HRP is half or less than that of PSG.

Published studies of the efficacy of HRP in comparison to conventional in-hospital PSG ${ }^{11-22}$ have shown positive results, but only four of them were randomised; ${ }^{14}{ }^{16-18}$ sample sizes were between 29 and 89 patients and there was a great deal of methodological variability. In addition, a detailed cost analysis for equivalent efficacy is essential for determining effectiveness and clinical applications of alternatives. However, only three studies, ${ }^{171820}$ included cost estimations. A multicentre design is another important factor for minimising inter-centre variability in costs, performance and scoring of sleep studies.

The HRP scoring produced lower AHIs than PSG. This could have been caused by longer recording times with HRP in comparison to PSG sleep time and some of the following factors: shorter sleep time in a supine position, ${ }^{13-15}$ night-to-night variability, and better sleep quality and stability, resulting in fewer obstructive events ${ }^{30}$ summarised by performing PSG and HRP on different nights. In fact, SRP had better agreement with PSG than HRP in the same patients. The remaining disagreement between PSG and SRP was similar to that seen in other studies and may primarily be caused by different equipment being used (different brand, models and software for displaying and analysing signals from the nasal cannula, thoracic and abdominal bands and, especially, the oximeter), inter-centre variability in identifying respiratory events, and also the inclusion of arousal in the PSG hypopnoea definition, although greater disagreement is not seen in studies including arousal ${ }^{14} 16$ 16-34 when compared with those without arousal. ${ }^{152122}$

The fact that PSG is carried out in 'unnatural' conditions has led to doubt as to whether PSG is the gold standard. Two randomised controlled studies have focused the results of CPAP treatment $\left(12\right.$ weeks $^{35}$ and 4 weeks $\left.{ }^{36}\right)$ after diagnosis of SAHS by PSG or home portable monitor (oximetry ${ }^{35}$ and oximetry, flow and body position ${ }^{36}$ ). Both studies showed similar improvements in AHI, quality of life, clinical symptoms and adherence to CPAP treatment. This study was focused in a different way: cost effectiveness was analysed in a large sample of patients using a multicentre approach. Thus, new, longer studies with HRP are needed, including collection of cardiovascular variables and cost. 
Table 2 Exclusionary and confirming HRP cut-off points with polysomnographic AHI cut-off points of $\geq 5, \geq 10$ and $\geq 15$

\begin{tabular}{|c|c|c|c|c|c|c|c|}
\hline & Selectivity & Specificity & $\mathrm{LR}+(95 \% \mathrm{CI})$ & Post-test probability+ $(95 \% \mathrm{Cl})$ & $\mathrm{LR}-(95 \% \mathrm{CI})$ & Post-test probability - $(95 \% \mathrm{CI})$ & $\mathrm{HRP}+/-, \%$ \\
\hline \multicolumn{8}{|c|}{ To AHI $\geq 5$ from PSG (pretest probability $90 \%$ ) } \\
\hline$\geq 5$ & 96 & 57 & 2.23 (1.78 to 2.79$)$ & 95 (94 to 96$)$ & $0.07(0.05$ to 0.10$)$ & 39 (31 to 47$)$ & $90 / 10$ \\
\hline$\geq 10$ & 87 & 86 & $6,25(2.73$ to 14$)$ & 98 (96 to 99$)$ & $0.15(0.11$ to 0.21$)$ & $57(50$ to 65$)$ & $80 / 20$ \\
\hline$\geq 5$ & 97 & 39 & 1.59 (1.30 to 1.94$)$ & $88(86$ to 90$)$ & $0.08(0.04$ to 0.16$)$ & 27 (15 to 42$)$ & $90 / 10$ \\
\hline$\geq 20$ & 71 & 90 & $7.10(3.37$ to 15$)$ & 97 (94 to 99$)$ & $0.32(0.26$ to 0.39$)$ & 59 (54 to 64$)$ & $60 / 40$ \\
\hline \multicolumn{8}{|c|}{ To $A H I \geq 15$ from PSG (pretest probability $75 \%$ ) } \\
\hline
\end{tabular}

AHI, apnoea-hypopnoea index; HRP, home respiratory polygraphy; HRP +/-, \%, percentage of patients with a positive/negative diagnosis based on HRP; LR, likelihood ratio; post-test probability + , the post-test probability of obtaining a true positive diagnosis when the test (HRP) was positive; post-test probability -, the post-test probability of obtaining a true positive diagnosis when the test (HRP) was negative.

If Mulgrew and Whitelaw's results are applied, that is, similar efficacy for HRP and PSG is assumed, the estimated diagnostic cost of HRP should be $€ 108$ (test cost for equal efficacy minus the cost of PSG in patients with indeterminate results-'grey zone'-and the cost of PSG in patients with false negative and false positive results) compared with $€ 554$ for PSG.

This cost analysis used the least favourable polysomnographic cut-off point, but when using the most favourable cut-off point with our results (polysomnographic $\mathrm{AHI} \geq 5$ ), the cost for equal efficacy decreased approximately $40 \%$ using two HRP cut-off points $(<5$ and $\geq 10)$ to rule out or confirm SAHS. Therefore, PSG costs would be roughly four times higher than HRP costs.

The costs of HRP and PSG can vary widely between countries and hospitals. In our consortium of hospitals, PSG costs varied between $€ 340$ and $€ 1074$, and HRP costs between $€ 65$ and $€ 175$. To a large extent, the favourable cost effectiveness of HRP was because of differences in test costs between PSG and HRP. Of three studies evaluating costs, ${ }^{17-19}$ two used both PSG and HRP. In one Scottish study ${ }^{19}$ the PSG cost was 4.6 times higher than the HRP cost, and in another from Spain ${ }^{18}$ (not from our consortium) the PSG cost was 2.6 times higher, but the cost of HRP included expenses for a nurse who visited patients at home and set up the device. Our ratio was higher (5.9). Even if the PSG/ $\mathrm{RP}$ cost ratio were decreased to that of the Scottish study (4.6), PSG would be $40 \%$ more expensive than HRP for equal efficacy.

The total HRP cost for equal diagnostic efficacy with the telematic approach was similar to non-telematic testing (when patients transported the device). The authors understand that this alternative procedure can be beneficial for patients with less mobility or those who live a long way from a hospital. The selection of patients in this study did not strictly exclude those with comorbidities as defined by AASM recommendations. ${ }^{9}$ The results indicate that, if moving patients can be avoided using

Table 3 Cost per patient of PSG and HRP (with and without telematic approach), for the different cost groups in the eight hospitals*

\begin{tabular}{|c|c|c|c|}
\hline & PSG & HRP & Telematic HRP \\
\hline Test cost & $540.60(183.51)$ & 91.35 (26.62) & $131.24(26.08)$ \\
\hline Patient cost & $22.21(69.62)$ & $44.42(69.62)$ & - \\
\hline Total cost & $562.81(186,51)$ & $135.77(67.06)$ & $131.24(26.08)$ \\
\hline $\begin{array}{l}\text { Cost for equal diagnostic } \\
\text { efficacy }\end{array}$ & $554.08(180.75)$ & $283.86(26.17)$ & $325.69(26.08)$ \\
\hline $\begin{array}{l}\text { Patient cost for equal } \\
\text { diagnostic efficacy }\end{array}$ & 23.13 (58.88) & $49.98(59.62)$ & - \\
\hline $\begin{array}{l}\text { Total cost for equal } \\
\text { diagnostic efficacy }\end{array}$ & $577.21(186.51)$ & $333.84(67.06)$ & $335.69(26.08)$ \\
\hline
\end{tabular}

a telematic approach to home diagnosis, initial exclusion of these patients may not be necessary. A study examining patients with chronic heart failure has shown that HRP has good diagnostic accuracy for SAHS, ${ }^{37}$ but more studies seem necessary.

The repetitions caused by the telematic approach were $7 \%$ of all recordings ( $37 \%$ of the total repetitions). Half of the telematic repetitions were caused by a failure in the data card initialisation in our prototype; this occurred with the initial patients and disappeared later. The outcome of the present study should be that new HRP devices should include a GPRS/UMTS emitter to simplify the process.

Some studies have estimated the costs of HRP compared with PSG based on simulated models of hypothetical cohorts of patients including diagnosis, CPAP titration and CPAP adherence, with contradictory results. ${ }^{23} 2438$ This study had a better methodological approach because it was carried out on a real, large cohort of patients, but the cost estimation was limited to diagnosis. Although the diagnostic method seems to be the most important factor for finding cost differences in these hypothetical cohort studies, rather than CPAP titration or CPAP adherence, the methodological differences may lead to poorly comparable results. This study and other similar ones ${ }^{17} 1820$ agree in concluding that HRP has a lower cost in comparison to PSG.

A limitation of the present study is the lack of centralised scoring for HRP and PSG, which would improve consistency. However, this is not commonly done in current clinical practice.

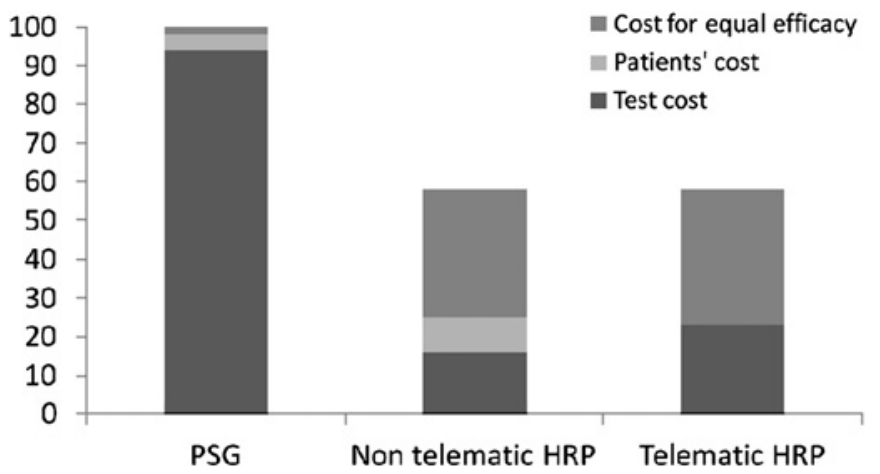

Figure 4 Percentages of total HRP costs and their distribution in three groups (test cost, patients' cost and cost for equal efficacy) compared with polysomnography costs, which is considered to be $100 \%$. Total HRP costs (telematic and non-telematic approaches) were roughly half the total cost of PSG. Costs for equal efficacy were more than half of the total HRP costs. In the telematic case, the test cost was similar to the sum of test costs and patients' costs in the non-telematic HRP approach. HRP, home respiratory polygraphy; PSG, polysomnography. 
Nevertheless, statistical differences were not found among centres for PSG and HRP AHI scorings.

Another issue is that the proportion of patients included in the study in relation to the total number of sleep clinic patients was not known. However, the prevalence of SAHS is similar to other studies assessing the diagnostic efficacy of HRP 1213151721 and the PSG AHI in this study was $38.3 \pm 28.5$. Therefore, the authors believe that the selection of patients represents a population with an intermediate or high probability of moderate to severe SAHS

A nasal cannula without an oral flow sensor was used in this study, which could overestimate obstructive events. However, the effect of this overestimation can be minimised because our consortium uses only nasal cannula in clinical practice and we are trained to identify oral leakage by the change in the wave shape from the nasal cannula. In addition, any potential bias would apply to both HRP and PSG.

In conclusion, HRP is a significantly lower cost alternative for diagnosis in patients with SAHS. Having devices moved by a transportation company and sending raw data telematically can be beneficial to patients with limited mobility or those who live a long way from the hospital.

Acknowledgements We are indebted to Verónica Rodríguez and Vanessa Iglesias for their assistance in the translation of the manuscript and Asunción Martín, Elena Sandoval, Soledad Guillen, Trinidad Amigo, Pablo Mejias, and Carmen Lorenzana for technical assistance in the sleep laboratory.

Funding Instituto de Salud Carlos III (Fondo de Investigaciones Sanitarias, Ministerio de Sanidad y Consumo), Spanish Respiratory Society (SEPAR), Telefonica SA (Spain), Air Liquide (Spain) and Breas Medical (Spain). We are grateful for the support of project EC02009-14152 (Ministerio de Ciencia e Innovación).

\section{Competing interests None.}

Patient consent Obtained.

Provenance and peer review Not commissioned; externally peer reviewed.

\section{REFERENCES}

1. Durán J, Esnaola S, Rubio R, et al. Obstructive sleep apnea-hypopnea and related clinical features in a population-based sample of subjects aged 30 to $70 \mathrm{yr}$. $\mathrm{Am} \mathrm{J}$ Respir Crit Care Med 2001;163:685-9.

2. Bazzano LA, Khan Z, Reynolds K, et al. Effect of nocturnal nasal continuous positive airway pressure on blood pressure in obstructive sleep apnea. Hypertension 2007:50:417-23.

3. Marin JM, Carrizo SJ, Vicente E, et al. Long-term cardiovascular outcomes in men with obstructive sleep apnoea-hypopnoea with or without treatment with continuous positive airway pressure: an observational study. Lancet 2005:365:1046-53.

4. Terán-Santos J, Jiménez-Gómez A, Cordero-Guevara J. The association between sleep apnea and the risk of traffic accidents. Cooperative Group Burgos-Santander. N Engl J Med 1999:340:847-51.

5. Masa JF, Rubio M, Findley LJ. Habitually sleepy drivers have a high frequency of automobile crashes associated with respiratory disorders during sleep. Am J Respir Crit Care Med 2000;162:1407-12.

6. Flemons WW, Douglas NJ, Kuna ST, et al. Access to diagnosis and treatment of patients with suspected sleep apnea. Am J Respir Crit Care Med 2004;169:668-72.

7. Masa Jiménez JF, Barbé Illa F, Capote Gil F, et al; Working Group. [Resources and delays in the diagnosis of sleep apnea-hypopnea syndrome] (In Spanish). Arch Bronconeumol 2007:43:188-98

8. Flemons WW, Littner MR, Rowley JA, et al. Home diagnosis of sleep apnea: a systematic review of the literature. An evidence review cosponsored by the American Academy of Sleep Medicine, the American College of Chest Physicians, and the American Thoracic Society. Chest 2003;124:1543-79.

9. Collop NA, Anderson WM, Boehlecke B, et al; Portable Monitoring Task Force of the American Academy of Sleep Medicine. Clinical guidelines for the use of unattended portable monitors in the diagnosis of obstructive sleep apnea in adult patients. Portable Monitoring Task Force of the American Academy of Sleep Medicine. J Clin Sleep Med 2007:3:737-47.

10. Ferber R, Millman R, Coppola M, et al. Portable recording in the assessment of obstructive sleep apnea. ASDA standards of practice. Sleep 1994:17:378-92.
11. Su S, Baroody FM, Kohrman M, et al. A comparison of polysomnography and a portable home sleep study in the diagnosis of obstructive sleep apnea syndrome. Otolaryngol Head Neck Surg 2004:131:844-50.

12. Redline S, Tosteson T, Boucher MA, et al. Measurement of sleep-related breathing disturbances in epidemiologic studies. Assessment of the validity and reproducibility of a portable monitoring device. Chest 1991;100:1281-6.

13. Yin $\mathbf{M}$, Miyazaki S, Ishikawa K. Evaluation of type 3 portable monitoring in unattended home setting for suspected sleep apnea: factors that may affect its accuracy. Otolaryngol Head Neck Surg 2006;134:204-9.

14. García-Díaz E, Quintana-Gallego E, Ruiz A, et al. Respiratory polygraphy with actigraphy in the diagnosis of sleep apnea-hypopnea syndrome. Chest 2007:131:725-32.

15. Ayappa I, Norman RG, Seelall V, et al. Validation of a self-applied unattended monitor for sleep disordered breathing. J Clin Sleep Med 2008:4:26-37.

16. Santos-Silva R, Sartori DE, Truksinas V, et al. Validation of a portable monitoring system for the diagnosis of obstructive sleep apnea syndrome. Sleep 2009:32:629-36.

17. Parra 0, García-Esclasans N, Montserrat JM, et al. Should patients with sleep apnoea/hypopnoea syndrome be diagnosed and managed on the basis of home sleep studies? Eur Respir J 1997:10:1720-4.

18. Alonso Alvarez M de L, Terán Santos J, Cordero Guevara J, et al. [Reliability of home respiratory polygraphy for the diagnosis of sleep apnea-hypopnea syndrome: analysis of costs] (In Spanish). Arch Bronconeumol 2008:44:22-8.

19. Whittle AT, Finch SP, Mortimore IL, et al. Use of home sleep studies for diagnosis of the sleep apnoea/hypopnoea syndrome. Thorax 1997;52:1068-73.

20. White DP, Gibb TJ, Wall JM, et al. Assessment of accuracy and analysis time of a novel device to monitor sleep and breathing in the home. Sleep 1995;18:115-26.

21. Dingli K, Coleman EL, Vennelle M, et al. Evaluation of a portable device for diagnosing the sleep apnoea/hypopnoea syndrome. Eur Respir J 2003;21:253-9.

22. Reichert JA, Bloch DA, Cundiff E, et al. Comparison of the NovaSom QSG, a new sleep apnea home-diagnostic system, and polysomnography. Sleep Med 2003;4:213-18.

23. Chervin RD, Murman DL, Malow BA, et al. Cost-utility of three approaches to the diagnosis of sleep apnea: polysomnography, home testing, and empirical therapy. Ann Intern Med 1999;130:496-505.

24. Reuveni H, Schweitzer E, Tarasiuk A. A Cost-effectiveness analysis of alternative at home or in laboratory technologies for the diagnosis of obstructive sleep apnea syndrome. Med Decis Making 2001;21:451-8.

25. Rechtschaffen A, Kales A, eds. A manual of standardized terminology and scoring system for sleep stages of human subjects. Los Angeles: Brain information service/ Brain research institute, University of California, 1968.

26. Anon. Sleep-related breathing disorders in adults: recommendations for syndrome definition and measurement techniques in clinical research; the report of an American Academy of Sleep Medicine Task Force. Sleep 1999:22:667-89.

27. Grupo Español de Sueño (GES). Consenso Nacional sobre el síndrome de apneashipopneas del sueño. Arch Bronconeumol 2005;41(Suppl 4):3-110.

28. Iber C, Ancoli-Israel S, Chesson AL, et al. The AASM manual for the scoring of sleep and associated events. Westchester, III: American Academy of Sleep Medicine (AASM), 2007

29. Drummond M, O'Brien B, Stoddart GL, et al. Methods for the economic evaluation of health care programmes. Oxford. Oxford University Press, 1997.

30. Stepnowsky CJ Jr, Orr WC, Davidson TM. Nightly variability of sleepdisordered breathing measured over 3 nights. Otolaryngol Head Neck Surg 2004;131:837-43.

31. Calleja JM, Esnaola S, Rubio R, et al. Comparison of a cardiorespiratory device versus polysomnography for diagnosis of sleep apnoea. Eur Respir $J$ 2002;20:1505-10.

32. Candela A, Hernández $L$, Asensio $S$, et al. [Validation of a respiratory polygraphy system in the diagnosis of sleep apnea syndrome] (In Spanish). Arch Bronconeumol 2005; 41:71-7.

33. Ballester E, Solans M, Vila X, et al. Evaluation of a portable respiratory recording device for detecting apnoeas and hypopnoeas in subjects from a general population. Eur Respir J 2000;16:123-7.

34. To KW, Chan WC, Chan TO, et al. Validation study of a portable monitoring device for identifying OSA in a symptomatic patient population. Respirology 2009:14:270-5.

35. Mulgrew AT, Fox N, Ayas NT, et al. Diagnosis and initial management of obstructive sleep apnea without polysomnography: a randomized validation study. Ann Intern Med 2007:146:157-66.

36. Whitelaw WA, Brant RF, Flemons WW. Clinical usefulness of home oximetry compared with polysomnography for assessment of sleep apnea. Am J Respir Crit Care Med 2005:171:188-93.

37. Quintana-Gallego E, Villa-Gil M, Carmona-Bernal C, et al. Home respiratory polygraphy for diagnosis of sleep-disordered breathing in heart failure. Eur Respir $J$ 2004:24:443-8.

38. Deutsch PA, Simmons MS, Wallace JM. Cost-effectiveness of split-night polysomnography and home studies in the evaluation of obstructive sleep apnea syndrome. J Clin Sleep Med 2006:2:145-53. 